\title{
On the Convergence of Planar Diagram Expansions
}

\author{
Gerard 't Hooft \\ Institute for Theoretical Physics, University of Utrecht, Princetonplein 5, P.O. Box 80.006, NL-3508 TA \\ Utrecht, The Netherlands
}

\begin{abstract}
Renormalizable quantum field theories whose perturbation expansions are described by planar Feynman diagrams only, such as $\mathrm{SU}(\infty)$ gauge theory, are considered in 4 dimensional Euclidean space. For studying asymptotic properties of the perturbation series one might wish to isolate first all those planar diagrams that do not contain any ultraviolet divergent subgraphs. In this paper it is proved that this infinite set of diagrams, when summed, converges within a finite radius of convergence for the coupling constant.
\end{abstract}

\section{Introduction}

In spite of considerable efforts is is still not known how to compute physical quantities accurately in any four dimensional quantum field theory with strong interactions. It seems quite likely that if any strong interaction field theory exists in which accurate computations can be done, then that must be an asymptotically free, non-Abelian gauge theory. In such theories the small-distance structure is completely described by the solutions of the renormalization group equations; and there are reasons to believe that the continuum theory can be uniquely defined as a limit of a lattice gauge theory, when the size of the meshes of the lattice tends to zero, together with the coupling constant, in a way prescribed by this renormalization group. Indeed one can prove using the formalism of [1] that this limit exists up to any finite order in the perturbation expansion for small coupling.

However, this result has not been extended beyond perturbation expansion. Therefore, the popular belief that theories such as "quantum chromodynamics" are mathematically well-founded might be preposterous. In [2] this author speculated that physical changes in the QCD theory might be necessary in order to tame its (suspected) disease: perhaps the number of colors $N$ has to tend to infinity at increasing energies. Arguments were presented that hold some promise that a mathematically accurate continuum field theory can be constructed along these lines, but much more work on the expansions in the effective coupling constant $\tilde{g}^{2}=g^{2} N$ must be done. 
A second, more traditional, reason to study the $\tilde{g}^{2}$ expansion at large $N$ is of course that this may be an interesting approximation for QCD in its own right, and if somebody can sum this expansion (which turns out to give only planar Feynman diagrams [3]) then that will yield a treasure of important information.

Our claim is now that at infinite $N$, the expansion in $\tilde{g}^{2}$ can be given a mathematically unique interpretation in 4 dimensions. We will not present a proof of that statement in this paper; the proof, which we sketched superficially in [2], is still postponed to a later publication. Here, we derive an intermediate result, necessary as a first step towards that proof.

The planar diagrams considered in this paper are only a subset of all diagrams: we take only those diagrams that contain no ultraviolet divergent subgraphs anywhere. This subset is large and important. Diagrams that do have divergent subgraphs can be added later. Divergent subgraphs must have four or less external legs and can therefore be seen as vertex- and propagator-insertions. So the diagrams that we leave out now do not much more than "renormalize" the vertices and propagators in the diagrams that we do consider here.

We prove in this paper that if the absolute value(s) of the coupling constant(s) $\tilde{g}^{2}$ is (are) small enough then the sum of all diagrams of this type converges uniformly ${ }^{1}$ in Euclidean space. At various stages of this proof planarity and UV finiteness of the diagrams considered are crucial. The theorem is non-trivial only because no further ultraviolet or infrared cut-off is required. It holds also for massless theories.

One of the main problems in setting up the proof turned out to be the bookkeeping: there are so many different kinds of planar diagrams to be considered. We try to keep it as simple and general as possible. The price, as we will see, is that often the required inequalities are satisfied with only a small margin, and this reduced the actual radius of our circle of convergence to a rather small value $\left(\sim 10^{-2}\right)$, mainly because with every step to make different diagrams look more alike we loose a few factors of 2 . With somewhat more effort it should be possible to raise this radius closer to the value one.

\section{The Diagrammatic Rules}

The details of the Feynman rules are to a large extent irrelevant for us. We make no use of cancellations among different diagrams due to sign differences; indeed, in momentum space we consider only the integrals over the absolute values of the original integrands. For instance, in the Yang-Mills theory the three-vertex carries as a factor

$$
g f^{a b c}(p-q)_{\mu} \delta_{v \lambda}+\text { permutations . }
$$

We could just as well replace it by

$$
g^{\prime}(|p|+|q|+|k|)
$$

where $p, q$, and $k=p+q$ are the 4-momenta of the external lines. Also the fermionic minus signs for the ghosts are dropped, so these ghosts are just treated as some

1 We mean to say that the convergence is uniform apart from some trivial overall multiplicative factors $\left[\left(k_{i}-k_{j}\right)^{2}+m^{2}\right]^{-1}$, where $k_{i}$ and $k_{j}$ are external momenta 
other "regular" field. We presume that the number of different kinds of fields after removal of the color indices which run from one to $N$, remains finite. Then we can simply absorb all such details, including Lorentz indices, into one coupling constant $g^{\prime}$. So all we are left with are the kinematical dependences on powers of the various momenta running through a diagram. Clearly then, our theorem will be valid just as much for planar $\phi^{4}$ theories as for gauge theories.

The vertex function (2.2) will be considered as a sum of three different vertex functions. Similarly there is a four-vertex which is just a constant. The propagator is $\left(k^{2}+m^{2}-i \varepsilon\right)^{-1}$. The value of $m$ is free and will turn out to be irrelevant. Note $m=0$ is allowed throughout; the mass term was added just because it does no harm to our arguments anywhere and might be convenient for other theories.

Since in (2.2)

$$
|p| \leqq\left(p^{2}+m^{2}\right)^{1 / 2}, \text { etc. },
$$

we may now limit ourselves to the following set of rules, which we will call type I rules for planar diagrams:

1) Propagators carry a factor $\left(k^{2}+m^{2}\right)^{-1}$.

2) Three-vertices carry a factor $\left(k^{2}+m^{2}\right)^{1 / 2}$, where $k$ is one of the momenta coming in.

3) Four-vertices carry a factor 1 .

4) The diagrams carry a factor $\left(g^{\prime}\right)^{V_{3}+2 V_{4}}$, where $g^{\prime}$ depends linearly on $\tilde{g}$.

$V_{3}$ is the number of 3-vertices.

$V_{4}$ is the number of 4-vertices.

5) Total 4-momentum is conserved at all vertices, and every closed loop (facet) corresponds to an integration $d^{4} k$ over the Euclidean momentum running through that loop. None of these integrals may diverge for large $k$. This implies that neither the diagram itself nor any of its subdiagrams may contain 4 or less external lines.

An amplitude is then defined by the sum of all different diagrams with a given number of external lines with given values of the external momenta.

We will show in Sect. 5 that the integrals corresponding to these type I diagrams are bounded by those of a slightly different set with different rules, which we refer to as type II planar diagrams. They differ from type I in the following way. Type II :

1) There are only 3 -vertices. They carry a factor 1 . Associated to them there are three coefficients $\alpha_{1}, \alpha_{2}, \alpha_{3}$, belonging to the three lines. At each vertex

$$
\alpha_{1}=\alpha_{2}=0, \quad \alpha_{3}=\frac{1}{2}
$$

or permutations thereof.

2) The propagators carry a factor

$$
\left|k^{2}+m^{2}\right|^{-\left(1+\alpha_{\imath}+\alpha_{3}\right) / 2},
$$

where $\alpha_{i}$ and $\alpha_{j}$ are the coefficients of both vertices connected by the propagator.

3) There are no subdiagrams with three external lines. The subdiagrams with four external lines have at least one of their external $\alpha$ coefficients equal to zero. This way the integrals inside these subdiagrams are guaranteed to converge.

The main step in our procedure will be to show that the integral of every type II diagram can be bounded by those of other type II diagrams with fewer vertices and 


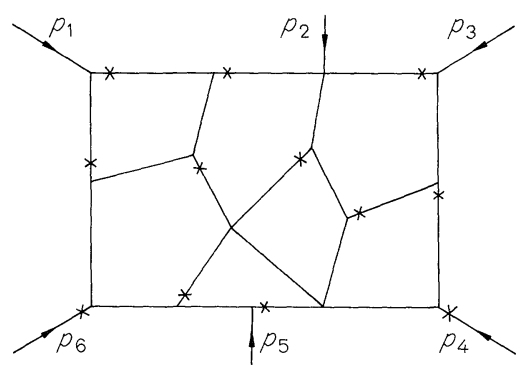

Fig. 1. Example of a type I diagram. At each 3-vertex a cross marks the extra momentum factor taken

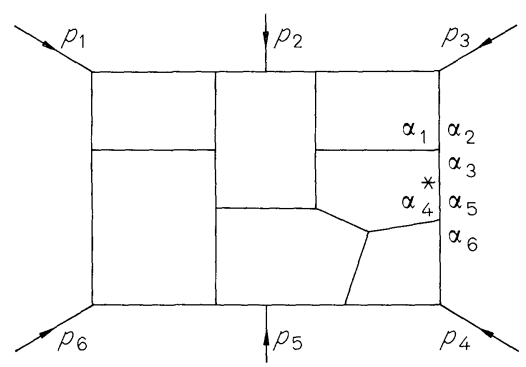

Fig. 2. Example of a type II diagram. We have: $\alpha_{1}+\alpha_{2}+\alpha_{3}=\alpha_{4}+\alpha_{5}+\alpha_{6}=\frac{1}{2}$, and the propagator marked with * carries a factor $\left(k^{2}+m^{2}\right)^{-\left(1+\alpha_{3}+\alpha_{5}\right) / 2}$

loops, multiplied with coefficients $C$ that do not exceed certain values regardless of the momentum values of the external lines.

\section{The Number of Type I Diagrams}

Any (planar or non-planar) diagram with $V_{3} 3$-vertices, $V_{4} 4$-vertices, $E$ external lines and $L$ loops satisfies

$$
V_{3}+2 V_{4}=E+2 L-2 \text {. }
$$

The number $L$ equals the number of facets in a planar diagram. We are interested in the case $L \rightarrow \infty$ at fixed $E$, so often we will ignore $E-2$ in (3.1).

The number of planar diagrams with given $E$ and $L$ grows exponentially with $L$, and not factorially as in non-planar theories [4]. So for every amplitude there is a certain type I diagram and a constant $C_{1}$ such that

$$
\left.A \leqq C_{1}^{L} D \quad \text { (for large } L\right),
$$

where $D$ is the integral corresponding to this single diagram. The value of $C_{1}$ has been computed for some cases in $[5,6]$ and depends on the details of the diagrammatic rules. For instance, in $\phi^{3}$ theory with self-energy insertions, $C_{1}=27 / 2$. Without self-energy insertions, $C_{1}=256 / 27$.

Our type I diagrams also certain $\phi^{4}$ vertices. This enhances $C_{1}$ somewhat. A bound can be given by regarding 4-vertices as pairs of 3-vertices with new "propagators" in between. This gives a factor of at most

$$
2^{V_{4}} \rightarrow 2^{L}
$$


for large $L$. Furthermore, the three terms in Eq. (2.2) triple the number of type I diagrams at each 3-vertex, giving a factor that increases with $L$ at most as

$$
9^{L} \text {. }
$$

Combining these arguments we find that the number of type I diagrams for an amplitude grows not faster than

$$
\left(18 C_{1}\right)^{L}
$$

for large $L$. This can easily be reduced to

$$
\left(10 C_{1}\right)^{L},
$$

but in this paper we shall not really try to economize on the coefficients needed.

We conclude that what remains to be proven is that any type I diagram with a given number of external lines at given momentum values does not increase faster than exponentially with $L$. We do that by reducing that problem to type II diagrams. But first we need some useful inequalities.

\section{Schwarz' Inequality in Diagrams}

Frequently we encounter the expression $\left(p^{2}+m^{2}\right)^{1 / 2}$, for which we will reserve the absolute value symbol:

$$
|p| \underset{\text { def }}{=} \sqrt{p^{2}+m^{2}}
$$

We then have (note that we always restrict ourselves to Euclidean space):

$$
\left|p_{1}+p_{2}\right| \leqq\left|p_{1}\right|+\left|p_{2}\right|
$$

(Schwarz' inequality). From this one easily deduces that if

then

$$
p_{3}=p_{1}+p_{2} ; \alpha \geqq 0 ; \beta \geqq 0,
$$

$$
\frac{1}{\left|p_{1}\right|^{\alpha}\left|p_{2}\right|^{\beta}} \leqq \frac{A(\alpha, \beta)}{\left|p_{1}\right|^{\alpha}\left|p_{3}\right|^{\beta}}+\frac{A(\beta, \alpha)}{\left|p_{3}\right|^{\alpha}\left|p_{2}\right|^{\beta}} \text {, }
$$

for all $p_{1}, p_{2}$.

Here

If $\alpha \leqq 1$ and $\beta \leqq 1$, then

$$
A(\alpha, \beta) \leqq 2^{\beta}
$$

$$
A(\alpha, \beta)=1
$$

Often we indicate a propagator or self-energy insertion of the form $|p|^{-\alpha}$ by a line with symbol $\alpha$ :

The inequality (4.3) for a three-vertex can then be represented diagrammatically (Fig. 3).
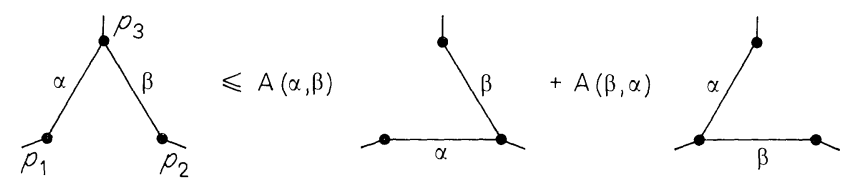

Fig. 3. Inequality (4.3) 
Another useful inequality is

$$
\frac{1}{\left|p_{1}\right|^{\alpha}\left|p_{2}\right|^{\beta}} \leqq \frac{1}{\left|p_{1}\right|^{\alpha+\beta}}+\frac{1}{\left|p_{2}\right|^{\alpha+\beta}} \text {. }
$$

simply because if $\left|p_{1}\right|<\left|p_{2}\right|$ the first term is sufficient, and otherwise the last.

\section{The Transition Towards Type II}

For bookkeeping purposes type II diagrams are handier because they only contain 3-vertices connected by propagators whose power of $|p|^{-1}$ is one or larger. As a start, we consider the 4-vertices in type I diagrams as pairs of 3-vertices connected with a propagator $|p|^{0}$. But each can be split in two ways. We use this freedom by splitting them in such a way that triangle subgraphs never occur. A triangle subgraph is a subgraph with only 3 external legs (two-leg subgraphs, "selfenergy insertions", would not occur anyhow because we assumed divergent subgraphs to be absent). It is not hard to check that this can always be done. Triangular subgraphs could only arise from UV convergent type I subgraphs with 5 or 6 external legs, coming from two or three 4-vertices. Only one of these needs to be split in the other direction (Fig. 4).

Of course one must check that no clashes occur if this requirement is considered for the different facets of the planar diagrams. But such a clash could only occur if, prior to the correction, diagram (b) of Fig. 4 would be surrounded by at least two 4-leg subgraphs, and that would turn the whole into a self-energy insertion, which was excluded from the beginning. Therefore it is always possible to split all 4-vertices avoiding any triangular subgraph.

Now we make use twice of inequality (4.3) at each 4-vertex, with $\alpha=\beta=\frac{1}{2}$. Here $p_{3}$ is the momentum through the new propagator, $p_{1}$ and $p_{2}$ are the two momenta at one side, and then at the other. We find the inequality sketched in Fig. 5:

$\left|p_{1}\right|^{-1 / 2}\left|p_{2}\right|^{-1 / 2}\left|q_{1}\right|^{-1 / 2}\left|q_{2}\right|^{-1 / 2} \leqq\left|p_{1}+p_{2}\right|^{-1}\left(\left|p_{1}\right|^{-1 / 2}+\left|p_{2}\right|^{-1 / 2}\right)\left(\left|q_{1}\right|^{-1 / 2}+\left|q_{2}\right|^{-1 / 2}\right)$,

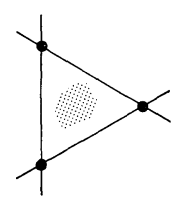

a

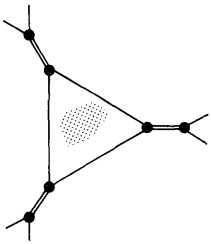

b

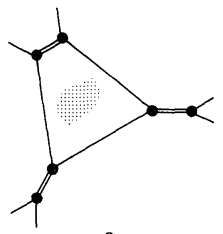

Fig. 4a-c. A type I subgraph a might produce a forbidden triangular subgraph after splitting the 4vertices $\mathbf{b}$. But only one 4-vertex needs be split the other way to avoid this $\mathbf{c}$. The shaded part represents possible further structure inside the triangle
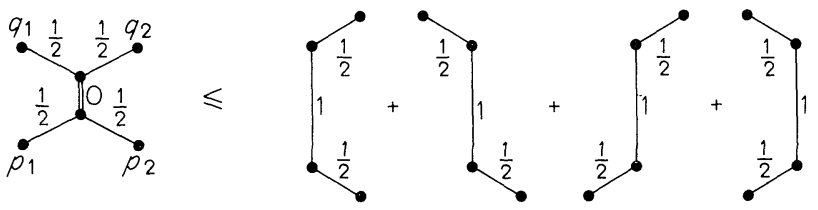

Fig. 5. The inequality (5.1) 
if

$$
p_{1}+p_{2}=q_{1}+q_{2}
$$

The 3-vertices in a type I diagram are now also subject to the inequality (4.3), again with $\alpha=\beta=\frac{1}{2}$. We write

$$
\left|p_{1}\right| \leqq\left|p_{1}\right|^{1 / 2}\left|p_{2}\right|^{1 / 2}+\left|p_{1}\right|^{1 / 2}\left|p_{3}\right|^{1 / 2}
$$

This way every type I diagram can be bounded by the sum of $2^{V_{3}+2 V_{4}} \propto 4^{L}$ diagrams which by inspection satisfy the specifications of type II. In particular note that our procedure also respects the third requirement: the inequalities never produce a 4-leg subgraph with all $\alpha$-coefficients sticking out being equal to $\frac{1}{2}$, because such a 4-graph could only have come after splitting one or more 4-vertices and as one can see in Fig. 5, their $\alpha$ coefficient in the new propagator equals zero. Therefore, the newly obtained type II diagrams are still all convergent.

The next step in our procedure will be to compare a type II diagram with type II diagrams whose number of facets $L$ has been reduced by one or more. For this we need inequalities relating a one loop graph with tree graphs. They are given in the next section.

\section{A Loop-Tree Inequality}

Clearly all one-loop subgraphs of type II diagrams are ultraviolet (and infrared) convergent. In this section we find either one or a sum of several tree graphs that may replace such a one-loop insertion, so that we can replace the loop by a tree. The newly obtained structure will not automatically satisfy all criterions for type II diagrams but that is the subject of Sects. 8 and 9 .

Consider the loop of Fig. 6 a.

It corresponds to the integral

$$
I=\int d^{4} k \prod_{i=1}^{n}\left[\left(k-p_{i}\right)^{2}+m^{2}\right]^{-\gamma_{i} / 2},
$$

where $p_{i}$ are determined by the external momenta. Those could be conveniently labeled as $p_{i i+1}=p_{i}-p_{i+1}$.

The tree graph in Fig. $6 \mathrm{~b}$ correponds to an expression of the form

$$
J=\prod_{i>j}\left[\left(p_{i}-p_{j}\right)^{2}+m^{2}\right]^{-\beta_{i j} / 2},
$$

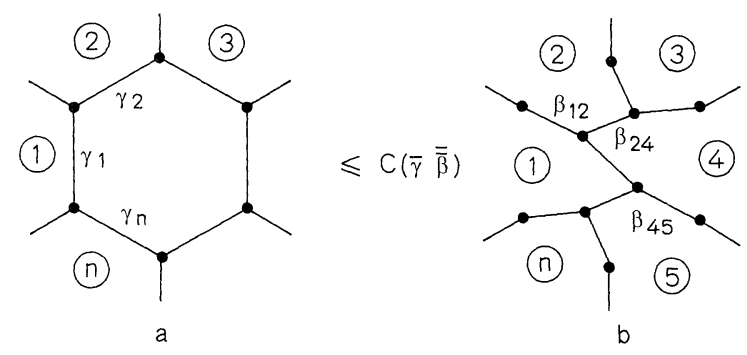

Fig. 6. Basic loop-tree inequality for an example with $n=6$ 
where the product is over all pairs of adjacent facets. Now the diagram and the numbers $\beta_{i j}$ must be chosen such that for all $i$

$$
\gamma_{i}>\sum_{j} \beta_{i j}
$$

All $\gamma_{i}$ and $\beta_{i j}$ must be positive. Furthermore,

$$
\sum \beta_{i j}=\sum_{i} \gamma_{i}-4
$$

for obvious dimensional reasons. Inequality (6.3) and Eq. (6.4) can only be satisfied if

$$
4<\sum_{i} \gamma_{i}<8
$$

Theorem. For any one-loop diagram satisfying inequality (6.5) one can find at least one (often several) tree diagrams with $\beta_{i j}$ satisfying (6.3) and (6.4).

The proof is simple but we will not need it because in all applications we construct the tree diagram explicitly.

Theorem. If (6.3)-(6.5) are fulfilled then there is a finite number $C(\vec{\gamma}, \overrightarrow{\vec{\beta}})$ such that for all values of $p_{i}$

$A$ bound for $C$ is

$$
I \leqq C(\vec{\gamma}, \overrightarrow{\vec{\beta}}) J
$$

$$
C(\vec{\gamma}, \vec{\beta}) \leqq \frac{\pi^{2} \Gamma\left(\sum \beta / 2\right)}{\Gamma\left(2-\sum \beta / 2\right)} \prod_{i} \frac{\Gamma\left(\left(\gamma_{i}-\sum_{j} \beta_{i j}\right) / 2\right)}{\Gamma\left(\gamma_{i} / 2\right)} \frac{\prod \beta_{i j}^{\beta_{i j} / 2}}{\left(\sum \beta\right)^{\Sigma / 2}} .
$$

The proof is straightforward using Feynman multiplicators. One finds

$$
I=\pi^{2} \frac{\Gamma\left(\sum \gamma / 2-2\right)}{\prod_{i} \Gamma\left(\gamma_{i} / 2\right)} \prod_{i} \int_{x_{\imath} \geqq 0} d x_{i} x_{i}^{\frac{\gamma_{i}}{2}-1} \frac{\delta\left(\sum x_{i}-1\right)}{\left(\sum_{i>j} Q_{i j} x_{i} x_{j}\right)^{\sum \gamma / 2-2}},
$$

where $Q_{i j} \geqq\left(p_{i}-p_{j}\right)^{2}+m^{2}$. [In fact the mass $m^{2}$ could be chosen larger: $m_{i}^{2}+m_{j}^{2}$, or in some cases even $\left(m_{i}+m_{j}\right)^{2}$.] Now we ask for the lowest possible value of

$$
R=\frac{\sum_{i>j} Q_{i j} x_{i} x_{j}}{\prod_{i>j}\left|Q_{i j}\right|^{e_{i j}}} \equiv \frac{D}{\prod},
$$

with

$$
\varrho_{i j}=\beta_{i j} / \sum \beta
$$

Extremizing with respect to one $Q_{i j}$ gives:

$$
\frac{\partial R}{R \partial Q_{i j}}=\frac{x_{i} x_{j}}{D}-\frac{\varrho_{i j}}{Q_{i j}}=0 \rightarrow Q_{i j}=\frac{\varrho_{i j} D}{x_{i} x_{j}} .
$$

The extreme value of $R$ is

$$
R_{\min }=\prod_{i>j} \varrho_{i j}^{-\varrho_{i j}} \prod_{i} x_{i}^{\sum_{j}^{j} \varrho_{i j}} .
$$


So

$$
I \leqq \frac{\pi^{2} \Gamma\left(\sum \gamma / 2-2\right)}{\prod_{i} \Gamma\left(\gamma_{i} / 2\right)} \prod_{i} \int d x_{i} x_{i}^{\frac{\gamma_{i}}{2}-1-\sum_{J} \beta_{t_{J} / 2}} \prod_{i>j} \beta_{i j}^{\beta_{i j} / 2}\left(\sum \beta\right)^{-\Sigma \beta / 2} \cdot J
$$

Integrating over the $x_{i}$ and remembering Eq. (6.4) we obtain (6.6) and (6.7).

Notice that an equal sign in inequality (6.3) would not be allowed because then $C \rightarrow \infty$, according to (6.7). There are special cases in which one can improve the theorem here, but we will not make use of that.

\section{Existence of Quadrangles and Pentagons}

Consider a one-particle irreducible planar graph. Euler's theorem for planar graphs is

$$
V-P+L=1,
$$

where $V=V_{3}+V_{4}$ is the number of vertices, $P$ is the number of propagators, and $L$ is the number of facets or loops. For type II diagrams, $V_{4}=0$.

Let $L=\sum_{n} F_{n}$, where $F_{n}$ is the number of facets with $n$ corners. Let $P=P_{i}+P_{e}$, where $P_{i}$ is the number of internal propagators and $P_{e}$ the number of propagators at the edges of the diagram. Then

$$
\sum_{n} n F_{n}=2 P_{i}+P_{e}
$$

Also

$$
\sum_{n} n V_{n}=3 V_{3}=2 P+E
$$

where $E$ is the number of external lines.

Combining (7.1)-(7.3) we find

$$
\sum_{n}(n-6) F_{n}=2 E-P_{e}-6 .
$$

Suppose that every planar irreducible subdiagram of our diagram had $2 E-P_{e} \geqq 6$. Then one can easily deduce that

$$
L \leqq \frac{E^{2}}{12}-\frac{E}{2}+1
$$

by applying induction with respect to $L$.

Since we are interested in $L \rightarrow \infty$ at fixed $E$, Eq. (7.5) cannot be fulfilled. Therefore according to Eq. (7.4) there must be at least one facet with $n=5$ or 4 . Usually there are many. Thus it should be possible to reduce the number of facets $L$ of any graph to a small number [satisfying (7.5)] by only applying the loop-tree theorem to quadrangles and pentagons ${ }^{2}$.

2 With an apology to linguists : words like "tetragons," "quintangles" etc. do not sound well 


\section{Quadrangles}

A general one-loop quadrangle diagram is shown in Fig. 7a. Its value can be limited by the integrals for two tree graphs, Fig. 7b and c. By assumption (type II diagrams) at least one $\alpha$ coefficient sticking out is zero, therefore at least one propagator goes with a power $\gamma \geqq 3 / 2$. The other $\gamma$ 's satisfy

$$
\gamma_{i} \geqq 1
$$

and all are $\gamma \leqq 2$, so that the integral is UV and IR convergent:

$$
Z=\sum \gamma_{i}-4 \geqq \frac{1}{2} \text {. }
$$

We also have $Z \leqq 2$.

Let us rotate or reflect the labels until

$$
\begin{aligned}
& \gamma_{2} \leqq \gamma_{4} \\
& \gamma_{1} \leqq \gamma_{3} .
\end{aligned}
$$

Now

$$
\gamma_{3}+\gamma_{4} \leqq 3 \frac{1}{2}
$$

therefore

$$
\gamma_{1}+\gamma_{2} \geqq Z+\frac{1}{2}
$$

so we can choose two coefficients $\lambda_{1,2} \geqq 0$ such that

$$
\begin{aligned}
& \lambda_{1}+\lambda_{2}=Z, \\
& \lambda_{1} \leqq \gamma_{1}, \\
& \lambda_{2} \leqq \gamma_{2}-\frac{1}{2}, \\
& \lambda_{1} \leqq Z-\frac{1}{2} .
\end{aligned}
$$

Now we use Eq.(4.3) and substitute in the integrand

$$
\frac{1}{\left|p_{1}\right|^{\lambda_{1}}\left|p_{3}\right|^{\lambda_{1}}} \leqq \frac{A\left(\lambda_{1} \lambda_{1}\right)}{\left|p_{1}-p_{3}\right|^{\lambda_{1}}}\left(\frac{1}{\left|p_{1}\right|^{\lambda_{1}}}+\frac{1}{\left|p_{3}\right|^{\lambda_{1}}}\right),
$$

where $p_{1}, p_{3}$ are the momenta in the loop as indicated in Fig. 7a. Here $p_{1}-p_{3}$ is a combination of external momenta. The two terms in the brackets yield diagrams which are less convergent:

$$
Z \rightarrow Z-\lambda_{1}=\lambda_{2} \geqq \frac{1}{2}
$$

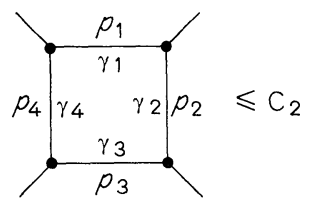

a

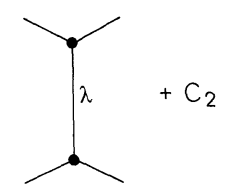

b

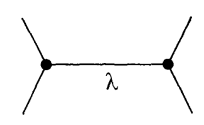

c

Fig. 7a-c. Inequality for quadrangles. The powers $\gamma$ and $\lambda$ for the propagators are explicitly indicated, as well as the flow of momenta $p$ 


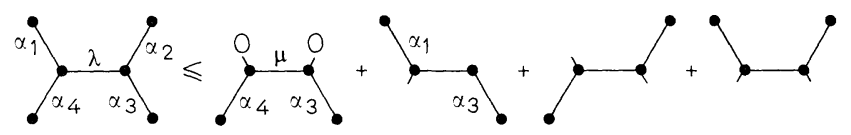

Fig. 8. Producing insertions for type II diagrams. The contributions of the $\alpha$ coefficients to the propagators are explicitly indicated. The short lines represent the absence of a propagator insertion

(The powers $\gamma_{1}$ and $\gamma_{3}$ are in turn replaced by $\gamma_{1}-\lambda_{1}$ and $\gamma_{3}-\lambda_{3}$.) For these new diagrams our loop-tree inequality is suitable. The inequalities (6.3) are all satisfied:

$$
\begin{aligned}
\lambda_{2}= & \beta_{24}<\gamma_{2}, \\
& \beta_{24}<\gamma_{4} .
\end{aligned}
$$

Our total diagram now satisfies

with

$$
I_{4} \leqq 2 \frac{A\left(\lambda_{1} \lambda_{1}\right)}{\left|p_{1}-p_{3}\right|^{\lambda_{1}}} \cdot C \cdot \frac{1}{\left|p_{2}-p_{4}\right|^{\lambda_{2}}}
$$

Finally we use Ineq. (4.4):

$$
C \leqq \frac{\pi^{2}\left(\Gamma\left(\frac{1}{4}\right)\right)^{3}}{\Gamma\left(2-\frac{1}{4}\right)}
$$

$$
\frac{1}{\left|p_{1}-p_{3}\right|^{\lambda_{1}}} \frac{1}{\left|p_{2}-p_{4}\right|^{\lambda_{2}}} \leqq \frac{1}{\left|p_{1}-p_{3}\right|^{\lambda}}+\frac{1}{\left|p_{2}-p_{4}\right|^{\lambda}}
$$

with $\lambda=\lambda_{1}+\lambda_{2}=Z$. Figure $7 \mathrm{~b}$ and $\mathrm{c}$ do not yet quite have the shape of a type II diagram. But we can again apply inequality (4.3) to obtain the diagram insertions of Fig. 8 .

The new propagators have

$$
\mu=2-\alpha_{4}-\alpha_{3} \geqq 1, \text { etc. },
$$

so that requirements 1) and 2) for type II diagrams are again met.

However, requirement 3) is not yet guaranteed: can triangles or divergent quadrangles emerge? First it is easy to prove that new divergent quadrangle (sub)graphs can always be avoided if they were not there from the beginning. If they would arise from one of the neighbouring pentagons changing into a quadrangle then one can always arrange that the new $\alpha$ parameter in the new propagator of Fig. $7 \mathrm{~b}$ and $\mathrm{c}$ vanishes, for instance by replacing $\left|p_{2}-p_{4}\right|^{\lambda_{2}}$ into

$$
\left|p_{2}-p_{4}\right|^{-\lambda_{2}^{\prime}}\left|p_{1}-p_{2}\right|^{-1 / 2}
$$

or

$$
\left|p_{2}-p_{4}\right|^{-\lambda_{2}^{\prime}}\left|p_{2}-p_{3}\right|^{-1 / 2}
$$

depending on which term in (8.7) in considered. No clash with type II requirements elsewhere arise.

Divergent composite 4-leg subgraphs do also not arise from manipulations in its subdiagrams, because (at least) one external $\alpha$-coefficient that is zero remains zero. 


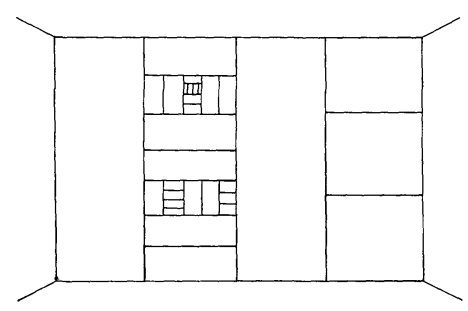

Fig. 9. Example of a generalized ladder graph

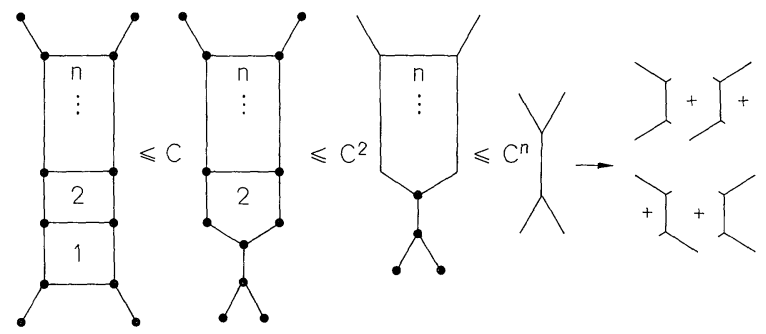

Fig. 10. Inequality for $n \geqq 2$ adjacent quadrangles

A tougher problem is that in some cases the transition quadrangle $\rightarrow$ tree is not allowed because a triangle could emerge as two of the adjacent subgraphs each lose an external leg. Suppose now that all fundamental quadrangles oppose application of the inequality of Fig. 7 because they are neighbours of at least one other elementary or composite quadrangular subgraph. The most general subgraph with interlocking quadrangles all having this pathological property may have the form of a "generalized ladder graph" (see Fig. 9). They consist of ladders inside ladders etc. Here it was also assumed that the subgraphs contain no pentagons that can be treated as in the next section.

Fortunately, the generalized ladder graphs can be handled quite easily, but by a slightly different procedure. We use inequalities of the form depicted in Fig. 10.

Consider two or more adjacent quadrangles. Take the first. We use the same method as before, but first, with equations just like (8.7), we split off factors

$$
\frac{1}{\left|p_{1}-p_{4}\right|^{\beta_{1}}} \text { and } \frac{1}{\left|p_{1}-p_{2}\right|^{\beta_{2}}}
$$

such that

$$
\begin{aligned}
& \beta_{1} \leqq \gamma_{4}, \\
& \beta_{2} \leqq \gamma_{2}, \\
& \beta_{1}+\beta_{2}=\max \left(Z-\frac{1}{4}, \gamma_{1}-\frac{1}{4}\right)
\end{aligned}
$$

The surviving diagram has $Z \geqq \frac{1}{4}$ and may be treated just as before. The factors (8.15) guarantee that whatever is left of the second quadrangle (which either remained a quadrangle or became a triangle) became more divergent with at most a factor $|k|^{1 / 4}$. So it has $Z \geqq \frac{1}{4}$. 

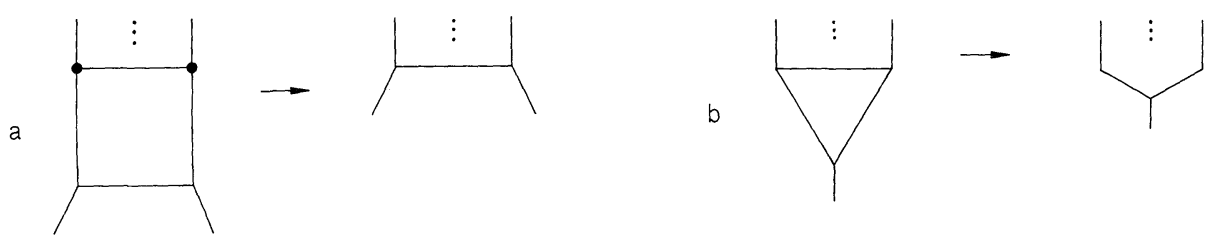

Fig. 11a and b. The second step of Fig. 10

The same procedure can now be repeated, except when the convergence coefficient $Z$ for the new diagram indeed becomes smaller than $\frac{1}{2}$. In that case the loop-tree inequality must be used exactly as in Fig. 11.

This way we are assured that $Z$ will never decrease below $\frac{1}{4}$. The final step in Fig. 10 is identical to the procedure sketched in Fig. 8. We can now repeat the inequality of Fig. 10 until the entire generalized ladder graph has been removed.

We end this procedure of removing quadrangles when no elementary quadrangles are left, or when composite quadrangle subgraphs consisting only of pentagons etc. survive. These subgraphs could possibly still be adjacent to elementary quadrangles because those quadrangles could not yet be treated. In all these cases we turn our attention to the elementary pentagons that now must exist, according to Sect. 7.

\section{Pentagons}

Again, in applying the loop-tree theorem to pentagons in type II diagrams, one special worry is tp obtain type II diagrams that satisfy also the third requirement. Now triangles will not emerge if quadrangles were not present before. If a composite 4-leg subgraph does occur then it might even sit next to elementary quadrangles to which the arguments of the previous section could not be applied. Then we consider this composite quadrangle first. Also in that case, we find pentagons in this composite quadrangle since it itself does not contain any elementary quadrangle. It is then easy to see that inside such a subgraph a pentagon can be found that is not adjacent to any quadrangle. It is this pentagon that can safely be removed by the following procedure; we do not have to worry about possible new (elementary or composite) quadrangles emerging, for which again the special convergence condition would have to be viewed.

We treat the pentagon essentially in the same way as the quadrangles in the beginning of Sect. 8 .

In this case

$$
\gamma_{i}=1+\alpha_{i 1}+\alpha_{i 2}
$$

are the total coefficients for the propagators. First we split off factors using inequality (4.3), for instance:

$$
\frac{1}{\left|k-p_{5}\right|^{\gamma_{5}}} \frac{1}{\left|k-p_{2}\right|^{\gamma_{2}}} \leqq \frac{A\left(\gamma_{5}, \gamma_{2}\right)}{\left|k-p_{5}\right|^{\mid \gamma_{5}}\left|p_{2}-p_{5}\right|^{\gamma_{2}}}+\frac{A\left(\gamma_{2}, \gamma_{5}\right)}{\left|p_{2}-p_{5}\right|^{\gamma_{5}}\left|k-p_{2}\right|^{\gamma_{2}}} \text {. }
$$

The two terms, inserted in the integral expression, give "quadrangle diagrams" because one propagator is eliminated, and to them the previous section applies. 
Fig. 12. The pentagon
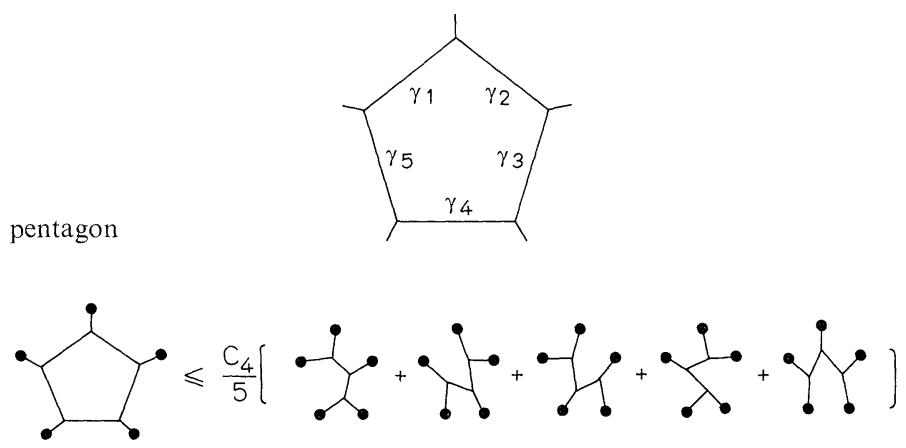

Fig. 13. Reduction of the pentagon

Now the following situation can be avoided by careful book-keeping, but one might worry that "impossible" external momentum dependence results :

$$
\frac{1}{\left|p_{2}-p_{5}\right|^{\gamma}} \frac{1}{\left|p_{1}-p_{4}\right|^{\lambda}}
$$

but this can be limited by

$$
\leqq \frac{1}{\left|p_{2}-p_{5}\right|^{\gamma+\lambda}}+\frac{1}{\left|p_{1}-p_{4}\right|^{\gamma+\lambda}}
$$

using inequality (4.4).

Finally, we use procedures similar to that depicted in Fig. 8 to obtain a set of genuine type II diagram insertions. Figure 13 results. Closer inspection shows that of the five tree diagrams only two are really necessary.

Counting factors, we find a limit for $C_{4}$ :

$$
C_{4} \leqq \frac{2^{8} \pi^{2} \Gamma\left(\frac{1}{4}\right)^{3}}{\Gamma\left(2-\frac{1}{4}\right)} \simeq \pi^{2} \cdot 2^{14} .
$$

Herewith we completed the proof that any type II diagram with a number of loops $L=L_{1}$ larger than $E^{2} / 12-E / 2+1$ [compare inequality (7.5)], is limited by the sum of a small set of type II diagrams with $L=L_{2}<L_{1}$ multiplied by some coefficient

$$
C^{L_{1}-L_{2}}
$$

and there ia an upper bound for $C$ :

$$
C \leqq \frac{C_{4}}{(2 \pi)^{4}} \leqq \frac{2^{10}}{\pi^{2}} \simeq 100,
$$

where the factor $(2 \pi)^{4}$ is the usual canonical one.

We did not attempt to keep $C$ small, so the actual bound one can derive from this paper is probably far larger than necessary. We feared that the much more extensive bookkeeping needed to keep $C$ small would make this paper rather untransparent. 


\section{Discussion}

The Green's functions considered were limited to Euclidean space [7]. We believe that this is not a serious flaw because the Euclidean amplitudes are usually believed to contain much if not all information about the real Minkowskian field theory. Of course, the Minkowskian amplitudes have poles and cuts and therefore convergence cannot be uniform in that region.

Our result would have been trivial if ultraviolet and infrared cutoffs were used. However, our theorems are valid without such cutoffs and also for massless theories. The danger was that at high orders there could have been a build-up of logarithms, and innocent-looking "convergent" integrals would develop factors $n$ ! in single diagrams [1]:

$$
\int \frac{d^{4} k\left[\log \left(k^{2}+Q\right)\right]^{n}}{\left(k^{2}+Q\right)^{3}}=\frac{\pi^{2} n !}{Q} \sum_{k=0}^{n} \frac{(\log Q)^{k}}{k !}\left(1-\left(\frac{1}{2}\right)^{n-k+1}\right) .
$$

This does happen in diagrams that were ultraviolet divergent and have been subtracted, see Fig. 14. Apparently such a thing does not happen in UVconvergent planar diagrams. Does it happen for non-planar UV-convergent diagrams? This we do not know. Our convergence proof breaks down because one can construct series of diagrams whose smallest oneloop subdiagrams have an increasing number of propagators. The number of such diagrams is also quite small however so our method can perhaps also be used there, despite the fact that $C$ coefficients relating arbitrarily large loops to tree diagrams are perhaps not uniformly bounded.

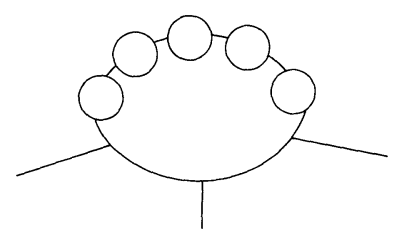

Fig. 14. Diagrams with subdivergences are, after subtraction of UV infinities, not geometrically bounded

\section{References}

1. 't Hooft, G.: In: The whys of subnuclear physics, Erice 1977, Zichichi, A. (ed.). New York, London: Plenum Press 1979, p. 943

2. 't Hooft, G.: Is asymptotic freedom enough? Phys. Lett. 109 B, 474 (1982)

3. 't Hooft, G.: A planar diagram theory for strong interactions. Nucl. Phys. B72, 461 (1974)

4. Dyson, F.: Divergence of perturbation theory in quantum electrodynamics. Phys. Rev. 85, 631 (1952)

Riddell, R.J., Jr.: The number of Feynman diagrams. Phys. Rev. 91, 1243 (1953)

5. Koplik, J., Neveu, A., Nussinov, S.: Some aspects of the planar perturbation series. Nucl. Phys. B123, 109 (1977)

6. Tuttle, W.T.: Can. J. Math. 14, 21 (1962)

7. Symanzik, K. : Euclidean quantum field theory. I. Equations for a scalar model. J. Math. Phys. 7, 510 (1966) 
8. De Calan, C., Rivasseau, V.: Local existence of the Borel transform in euclidean $\Phi_{+}^{4}$. Commun. Math. Phys. 82, 69 (1981)

9. Rivasseau, V.: Talk presented at the Conference on Statistical Mechanics and Quantum Field Theory, Rutgers University, USA, May 1980 (unpublished)

Communicated by A. Jaffe

Received March 23, 1982

Note added in proof: The author was informed that the methods of De Calan and Rivasseau [8] can be applied to reproduce his result, even though the theorems stated in their paper primarily deal with massive theories only. Our present formalism is quite different and will be developped further. The question raised in the end of this paper can be answered: also non-planar convergent diagrams are bounded geometrically [9], without mass-dependence. 